\title{
Comparison of Clinically Approved Molecules on SARS-CoV-2 Drug Target \\ Proteins: A Molecular Docking Study \\ Hasan Cubuk ${ }^{a}$ and Mehmet Ozbilb*
}

aDepartment of Molecular Biology and Genetics, Istanbul Arel University, 34537, Buyukcekmece, Istanbul.

'Institute of Biotechnology, Gebze Technical University, 41400, Gebze, Kocaeli.

*Corresponding author: Mehmet Ozbil, Institute of Biotechnology, Gebze Technical University, 41400, Gebze, Kocaeli.

Phone: +902626051376-2507 E-mail: mozbil@gtu.edu.tr

\section{Abstract}

The new type of coronavirus, SARS-CoV-2 has affected more than 6.3 million people worldwide. Since the first day the virus has been spotted in Wuhan, China, there are numerous drug design studies conducted all over the globe. Most of these studies target the receptor-binding domain of spike protein of SASR-CoV-2, which is known to bind human ACE2 receptor and SARS-CoV-2 main protease, vital for the virus' replication. However, there might be a third target, human furin protease, which cleaves the virus' S1-S2 domains taking active role in its entry into the host cell. In this study we docked five clinically used drug molecules, favipiravir, hydroxychloroquine, remdesivir, lopinavir, and ritonavir onto three target proteins, receptor binding domain of SARS-CoV-2 spike protein, SARS-CoV-2 main protease, and human furin protease. Results of molecular docking simulations revealed that human furin protease might be targeted against COVID-19. Remdesivir, a nucleic acid derivative, strongly bound to the active site of this protease, suggesting this molecule can be used as a template for designing novel furin protease inhibitors to fight with the disease. Protein-drug interactions revealed at the molecular level in this study can pave the way for better drug design for each specific target.

Keywords: SARS-CoV-2, COVID-19, Furin Protease, Remdesivir, Molecular Docking 


\section{Introduction}

Coronaviruses are a type of single-stranded RNA viruses that infect mammals and birds. In humans, they cause respiratory diseases ranging from common cold to severe/fatal illnesses. ${ }^{1}$ Three types of human-infecting coronaviruses were associated with deadly phenomenia since the early period of 2000 s; severe acute respiratory syndrome coronavirus (SARS-CoV), Middle-East respiratory syndrome coronavirus (MERS-CoV) and severe acute respiratory syndrome coronavirus 2 (SARS-CoV2). In November 2002, SARS-CoV affected 8,098 people and causing 774 deaths in China until the June 2003. In June 2012, MERS-CoV appeared in Middle East, over 2,000 cases and reported by 2017 with about 600 deaths. ${ }^{2-3}$

SARS-CoV-2, discovered in China, has affected over 6,100,000 people and killed 371,000 in more than 215 countries as of June 1, 2020. ${ }^{4}$ The World Health Organization (WHO) announced "COVID-19" as the name of new disease caused by SARS-CoV-2. The on going COVID-19 threat rapidly spread all over the globe and still transfecting humans. Thus, many efforts have been directed to the investigation of suitable preventive and control strategies in a few months as neither vaccines nor direct-acting antiviral drugs are available for the treatment of human SARS-CoV-2.

Most of the therapeutic options for COVID-19 were based on anti-viral agents, which are used for treating previous Zika, Ebola, and Nipah viruses, SARS-, and MERSCoVs. ${ }^{5}$ This is due to fact that the time required for drug discovery programs to develop, evaluate, and obtain appropriate new therapeutic agents might take more than 10 years. Thus, researchers are focused on therapeutics, which have proven efficacy against viruses similar to COVID-19 instead of a new potent anti-COVID-19 agent. These available therapeutic agents against SARS-CoV-2 could be either virus-based, involving small molecules targeting viral $S$ protein, viral protease inhibitors and RBD-ACE2 blockers or host cell-based including host cell protease inhibitors and host cell endocytosis inhibitors. ${ }^{5}$

Spike protein directly mediates viral entry with $\mathrm{S} 1$ domain, which is responsible for host cell surface binding through ACE2 receptors and S2 domain responsible for membrane fusion. The viral binding to host cell surface is following S1/S2 cleavage by host proteases such as TMPRSS2, cathepsins B and L. Previous studies have already demonstrated that furin, a proprotein convertase, can mediate S1/S2 
cleavage unlike other coronaviruses and contribute to membrane fusion efficiency which explain current strong infectious capacity of SARS-CoV-2.-3 Thus, SARSCoV-2RBD/ACE2 and Furin could be potential targets for COVID-19 to prevent viral entry. Furthermore, SARS-CoV-2 main protease known as $3 \mathrm{CL}^{\text {pro }}$ which is essential in processing viral polyproteins and viral replication could be a non-toxic target for managing COVID-19 as humans do not have proteases with similar cleavage specificity. ${ }^{1-2,6}$

In vitro studies by Liu et al. had already demonstrated that two drugs, chloroquine (CQ) and hydrochloroquine (HCQ) efficiently inhibited SARS-CoV-2 infection in vitro and these findings were supported by preliminary clinical studies as well. ${ }^{7-10}$ Several other drugs such as, remdesivir, and favipiravir are currently undergoing clinical studies to test their efficacy and safety in the treatment of COVID-19 in China and other European countries such as Turkey and some promising results have been achieved so far. ${ }^{11-15}$ In addition, lopinavir, and ritonavir are widely used as HIV protease inhibitors, and previous in vitro and in vivo studies have also shown their potential activity against other coronaviruses; SARS-, and MERS-CoVs. ${ }^{16-19}$

In the present study, we investigated binding of five active molecules, currently applied as the first line of treatment, favipiravir, hydroxychloroquine, remdesivir, lopinavir, and ritonavir onto three different possible target proteins, receptor binding domain of SARS-CoV-2 spike protein (SARS-CoV-2 ${ }^{R B D}$ ), SARS-CoV-2 main protease (SARS-CoV-2 Mpro), and human furin (hFUR) protease by molecular docking simulations. Our aim was to shed light on the functional group selection for future drug design studies by reporting protein-drug interactions at the molecular level.

\section{Methods}

\section{Preparation of the protein and target molecules}

The crystal structure of the human proprotein convertase furin (PDB: 1P8J, $2.6 \AA$ ),${ }^{20}$ COVID-19 main protease in complex with an inhibitor N3 (PDB: 6LU7, $2.2 \AA$ ), ${ }^{21}$ and coronavirus spike receptor-binding domain complexed with its receptor ACE2 (PDB: 6LZG, $2.5 \AA)^{22}$ were obtained from the Research Collaboratory for Structural Bioinformatics Protein Data Bank (RCSB PDB). Small molecules were removed from 
crystal structures by using BIOVA Discovery Studio software. ${ }^{23}$ Polar hydrogens and Kollman charges were added to the protein and a pdbqt format file was generated by using AutoDockTools 1.5.6 software. ${ }^{24}$

The canonical SMILES of Lopinavir, Remdesivir, Hydrochloroquine, Favipiravir and Ritonavir were obtained from PubChem database. Their structures were built, and structural optimization was carried out with USCF Chimera software. ${ }^{25}$ Afterwards, the structures were converted into pdbqt format by using AutoDockTools 1.5.6 software, in use for docking calculations with Vina.

\section{Docking}

Autodock Vina 1.1.2 software s6 $^{26}$ was used for docking calculations and exhaustiveness parameter was selected as 8 , and 10 modes were generated for each ligand. Windows 7 Ultimate operating system (64-bit) installed on a home-built desktop computer, equipped with Intel Core i3-3110M 2.40GHz processor and 8GB memory, was utilized for all computational work. Results were analysed using BIOVA Discovery Studio software and VMD-Visual Molecular Dynamics software. ${ }^{27}$

\section{Results and Discussion}

Due to the recent COVID-19 pandemia, many efforts have been directed to the investigation of suitable preventive and control strategies for for the treatment of human SARS-CoV-2 infection. Almost all these attempts are on the previously used anti-viral agents, such as lopinavir, ritonavir, favipiravir, hydroxychloroquine, and remdesivir. To unravel mechanism and inhibition capability of these five clinically used drugs against SARS-CoV-2 infections, we have run molecular simulations using three therapeutic targets; SARS-CoV-2 ${ }^{\text {RBD }}-A C E 2$ interface, SARS-CoV-2 Mproac and hFUR.

First, we designed docking simulations with grid box covering only SARS-CoV-2 ${ }^{\mathrm{RBD}}$ ACE2 interface. Binding of five molecules onto SARS-CoV-2RBD yielded binding affinities ranging from $-4.2 \mathrm{kcal} / \mathrm{mol}$ to $-6.9 \mathrm{kcal} / \mathrm{mol}$ (Table 1 and Figure 1 ). Among these active molecules lopinavir and ritonavir yielded higher binding affinities towards this target, $-6.9 \mathrm{kcal} / \mathrm{mol}$ and $-6.4 \mathrm{kcal} / \mathrm{mol}$, respectively. Favipiravir, hydroxychloroquine, and remdesivir were not potential binders of the protein. Relatively small sizes of favipiravir and hydroxychloroquine must be the reason for 
small binding affinities obtained in the study (Figure 2). Moreover, recent studies demonstrated that these drugs may target the inhibition of replication of viral genome and viral assembly. ${ }^{16}$ As we have shown in our data, these drugs are less likely to bind and inhibit SARS-CoV-2 ${ }^{\text {RBD }}$-ACE2 interface during SARS-CoV-2 infection.

Secondly, we set out docking simulations to examine binding of these drugs on SARS-CoV-2 Mpro, another very important element in COVID-19 infection. Docking analysis onto SARS-CoV-2 Mpro didn't yield significantly higher binding affinities than those for SARS-CoV-2RBD, ranging between from $-5.2 \mathrm{kcal} / \mathrm{mol}$ to $-6.6 \mathrm{kcal} / \mathrm{mol}$. Lopinavir was bound with the highest binding affinity, i.e. $-6.6 \mathrm{kcal} / \mathrm{mol}$ (Table 1 and Figure 1). All the binding sites for all five drug molecules were outside the active site of the protease. Therefore, we repeated simulations with smaller simulations box and this time targeting only the active site, SARS-CoV-2 Mpro-ac, comprised of amino acid residues Thr 26, His 41, Met 49, Leu 141, Asn 142, Gly 143, Gly 143, Ser 144, Met 165, Glu 166, and GIn 189. Molecular interactions at this site were provided in Figure 3. This time binding affinities increased for all ligands except for favipiravir. Specifically, for lopinavir and remdesivir binding affinities increased by $0.9 \mathrm{kcal} / \mathrm{mol}$ and $2.0 \mathrm{kcal} / \mathrm{mol}$, respectively. Both active molecules produced binding affinities of $7.5 \mathrm{kcal} / \mathrm{mol}$. Lopinavir is an antiretroviral protease inhibitor, approved by FDA. Our data suggests that lopinavir may also target SARS-CoV-2 $M^{\text {pro }}$ as it is and antiretroviral protease inhibitor of HIV-1. Hence, this would be an explanation for the clinical activity of lopinavir against SARS-CoV-2. This binding site was also revealed in the x-ray structure (PDB ID: 6LUV) ${ }^{21}$ for a peptide derivative inhibitor N3 and was also predicted to be lopinavir binding site in a computational study by Liu et.al. (Figure SI-1). ${ }^{19}$

The last target protein investigated was hFUR. Binding affinities obtained were in the range of $-6.0 \mathrm{kcal} / \mathrm{mol}$ to $-8.0 \mathrm{kcal} / \mathrm{mol}$ (Table 1 and Figure 1 ). Lopinavir provided the highest binding affinity with $-7.5 \mathrm{kcal} / \mathrm{mol}$ among all active molecules. On the other hand, remdesivir and ritonavir bound to this target with binding affinities $-6.6 \mathrm{kcal} / \mathrm{mol}$ and $-6.9 \mathrm{kcal} / \mathrm{mol}$, respectively. Furthermore, remdesivir was the only active molecule to hit the active site directly, even though the grid box covered the whole protein (Figure SI-2). We repeated docking simulations with a smaller grid box covering only active site of human furin ( $h F U R^{a c}$ ), comprised of amino acid residues 
Asp 153, Arg193, His 194, Arg 197, Leu 227, Val 231, Ser 253, Asp 258, and Asn

295. Molecular interactions were provided in Figure 4. Binding affinity for remdesivir increased by $0.4 \mathrm{kcal} / \mathrm{mol}$, while decreased for favipiravir by $0.9 \mathrm{kcal} / \mathrm{mol}$, hydroxychloroquine by $0.8 \mathrm{kcal} / \mathrm{mol}$, lopinavir by $1.2 \mathrm{kcal} / \mathrm{mol}$ and ritonavir by 0.1 $\mathrm{kcal} / \mathrm{mol}$, when compared to docking on whole protein structure. The only increase in binding affinities was observed for remdesivir upon covering only the active site of the protein. Highly hydrophilic and aromatic character of this pocket led to high binding affinity (Figure SI-3). This suggests that hFUR could be a main target for the remdesivir even if it involves in the inhibition of replication of viral genome and viral assembly. We should also mention that when all molecules were docked onto the whole hFUR, four molecules, favipiravir, hydroxychloroquine, ritonavir, and lopinavir didn't hit the active site. This suggested that hFUR ${ }^{a c}$ may not be accessible to these molecules, but to remdesivir.

\section{Conclusions}

In this study we investigated binding of readily prescribed drug molecules favipiravir, hydroxychloroquine, remdesivir, lopinavir, and ritonavir onto three therapeutic targets which should be targeted for COVID-19 treatment. Among all targets, receptor binding domain of SARS-Cov2 spike protein (SARS-CoV-2 ${ }^{\mathrm{RBD}}$ ), SARS-CoV-2 main protease (SARS-CoV-2 Mpro), and human furin (hFUR) protease, binding affinities for all drug molecule were calculated to be higher for active sites of both hFUR and SARS-CoV-2 Mpro. Moreover, remdesivir directly hit the active site of hFUR, with a binding affinity, $-7.0 \mathrm{kcal} / \mathrm{mol}$, while other drugs did not target the active site of $\mathrm{hFUR}$ directly. Reason for this direct targeting is the adenosine triphosphate moiety of remdesivir, making strong hydrophobic and polar interactions in the active site supported by hydrogen bonds.

Clinically used drug molecules; lopinavir, remdesivir, and ritonavir yielded higher binding affinities for both active sites of hFUR and SARS-CoV-2 Mpro than other therapeutic target; SARS-CoV-2 ${ }^{\mathrm{RBD}}$ while hydroxychloroquine and favipiravir yielded less affinity for these therapeutic targets by molecular docking simulations. In addition, remdesivir was a strong binder of the active site of hFUR. Thus, this should be major reason for potential activity of remdesivir against COVID-19 in the preclinical studies. hFUR could be targeted for the treatment of SARS-CoV-2 
infections other than usual drug targets such as SARS-CoV-2 Mpro and SARS-CoV$2^{\mathrm{RBD}}$. Moreover, remdesivir could be targeting both viral replication machines and host proteases responsible for the SARS-CoV-2 entry into cells. Our results cleary indicated that in order to design hFUR ${ }^{\text {ac }}$ inhibitors, remdesevir, a nucleic acid derivative, should be used as a template. Likewise, to target SARS-CoV-2 ${ }^{\mathrm{RBD}}$ or SARS-CoV-2 Mro, lopinavir like drug molecule should be designed. These findings will lead to more target secific drug design studies. 


\section{References}

1. Fung, T. S.; Liu, D. X., Human Coronavirus: Host-Pathogen Interaction. Annu Rev Microbiol 2019, 73, 529-557.

2. Canrong WU, Y. Y., Yang LIU, Peng ZHANG, Yali WANG, Qiqi WANG, Yang XU, Mingxue LI, Mengzhu ZHENG, Lixia CHEN, Hua LI Furin, a potential therapeutic target for COVID-19. 2020.

3. Walls, A. C.; Park, Y. J.; Tortorici, M. A.; Wall, A.; McGuire, A. T.; Veesler, D., Structure, Function, and Antigenicity of the SARS-CoV-2 Spike Glycoprotein. Cell 2020.

4.

https://covid19.who.int/?gclid=CjwKCAjw8df2BRA3EiwAvfZWaP34yJr8HdK4mBed5dKa2T6fl ZjBA5sFDNCata6LM6-eXa1CmMjHwhoCUZQQAvD BwE.

5. $\quad$ Dhama, K.; Sharun, K.; Tiwari, R.; Dadar, M.; Malik, Y. S.; Singh, K. P.; Chaicumpa, W., COVID19 , an emerging coronavirus infection: advances and prospects in designing and developing vaccines, immunotherapeutics, and therapeutics. Hum Vaccin Immunother 2020, 1-7.

6. Zhang, L.; Lin, D.; Sun, X.; Curth, U.; Drosten, C.; Sauerhering, L.; Becker, S.; Rox, K.; Hilgenfeld, R., Crystal structure of SARS-CoV-2 main protease provides a basis for design of improved $\alpha$-ketoamide inhibitors. Science 2020, eabb3405.

7. Liu, J.; Cao, R.; Xu, M.; Wang, X.; Zhang, H.; Hu, H.; Li, Y.; Hu, Z.; Zhong, W.; Wang, M., Hydroxychloroquine, a less toxic derivative of chloroquine, is effective in inhibiting SARS-CoV-2 infection in vitro. Cell Discov 2020, 6, 16.

8. Gao, J.; Tian, Z.; Yang, X., Breakthrough: Chloroquine phosphate has shown apparent efficacy in treatment of COVID-19 associated pneumonia in clinical studies. Biosci Trends 2020, 14 (1), $72-73$.

9. Wang, M.; Cao, R.; Zhang, L.; Yang, X.; Liu, J.; Xu, M.; Shi, Z.; Hu, Z.; Zhong, W.; Xiao, G., Remdesivir and chloroquine effectively inhibit the recently emerged novel coronavirus (2019-nCoV) in vitro. Cell Res 2020, 30 (3), 269-271.

10. Yao, X.; Ye, F.; Zhang, M.; Cui, C.; Huang, B.; Niu, P.; Liu, X.; Zhao, L.; Dong, E.; Song, C.; Zhan, S.; Lu, R.; Li, H.; Tan, W.; Liu, D., In Vitro Antiviral Activity and Projection of Optimized Dosing Design of Hydroxychloroquine for the Treatment of Severe Acute Respiratory Syndrome Coronavirus 2 (SARS-CoV-2). Clin Infect Dis 2020.

11. Dong, L.; Hu, S.; Gao, J., Discovering drugs to treat coronavirus disease 2019 (COVID-19). Drug Discov Ther 2020, 14 (1), 58-60.

12. https://khgmstokyonetimidb.saglik.gov.tr/TR, f.-.-m.-t.---.-c.---s.-c.-e.-t.-k.-i.-i.-b. h., Turkey.

13. Agostini, M. L.; Andres, E. L.; Sims, A. C.; Graham, R. L.; Sheahan, T. P.; Lu, X.; Smith, E. C.; Case, J. B.; Feng, J. Y.; Jordan, R.; Ray, A. S.; Cihlar, T.; Siegel, D.; Mackman, R. L.; Clarke, M. O.; Baric, R. S.; Denison, M. R., Coronavirus Susceptibility to the Antiviral Remdesivir (GS-5734) Is Mediated by the Viral Polymerase and the Proofreading Exoribonuclease. mBio 2018, 9 (2).

14. Brown, A. J.; Won, J. J.; Graham, R. L.; Dinnon, K. H., 3rd; Sims, A. C.; Feng, J. Y.; Cihlar, T.; Denison, M. R.; Baric, R. S.; Sheahan, T. P., Broad spectrum antiviral remdesivir inhibits human endemic and zoonotic deltacoronaviruses with a highly divergent RNA dependent RNA polymerase. Antiviral Res 2019, 169, 104541.

15. Ko, W. C.; Rolain, J. M.; Lee, N. Y.; Chen, P. L.; Huang, C. T.; Lee, P. I.; Hsueh, P. R., Arguments in favour of remdesivir for treating SARS-CoV-2 infections. Int J Antimicrob Agents 2020, 105933.

16. Tim Smith, P., BCPS; Jennifer Bushek, PharmD; Tony Prosser, PharmD, COVID-19 Drug Therapy - Potential Options. Elsevier 2020.

17. Chu, C. M.; Cheng, V. C.; Hung, I. F.; Wong, M. M.; Chan, K. H.; Chan, K. S.; Kao, R. Y.; Poon, L. L.; Wong, C. L.; Guan, Y.; Peiris, J. S.; Yuen, K. Y., Role of lopinavir/ritonavir in the treatment of SARS: initial virological and clinical findings. Thorax 2004, 59 (3), 252-6.

18. Chen, F.; Chan, K. H.; Jiang, Y.; Kao, R. Y.; Lu, H. T.; Fan, K. W.; Cheng, V. C.; Tsui, W. H.; Hung, I. F.; Lee, T. S.; Guan, Y.; Peiris, J. S.; Yuen, K. Y., In vitro susceptibility of 10 clinical isolates of SARS coronavirus to selected antiviral compounds. J Clin Virol 2004, 31 (1), 69-75. 
19. Liu, X.; Wang, X. J., Potential inhibitors against 2019-nCoV coronavirus M protease from clinically approved medicines. J Genet Genomics 2020.

20. Henrich, S.; Cameron, A.; Bourenkov, G. P.; Kiefersauer, R.; Huber, R.; Lindberg, I.; Bode, W.; Than, M. E., The crystal structure of the proprotein processing proteinase furin explains its stringent specificity. Nat Struct Biol 2003, 10 (7), 520-6.

21. Jin, Z.; Du, X.; Xu, Y.; Deng, Y.; Liu, M.; Zhao, Y.; Zhang, B.; Li, X.; Zhang, L.; Peng, C.; Duan, Y.; Yu, J.; Wang, L.; Yang, K.; Liu, F.; Jiang, R.; Yang, X.; You, T.; Liu, X.; Yang, X.; Bai, F.; Liu, H.; Liu, X.; Guddat, L. W.; Xu, W.; Xiao, G.; Qin, C.; Shi, Z.; Jiang, H.; Rao, Z.; Yang, H., Structure of M(pro) from SARS-CoV-2 and discovery of its inhibitors. Nature 2020.

22. Wang, Q.; Zhang, Y.; Wu, L.; Niu, S.; Song, C.; Zhang, Z.; Lu, G.; Qiao, C.; Hu, Y.; Yuen, K.-Y.; Wang, Q.; Zhou, H.; Yan, J.; Qi, J., Structural and Functional Basis of SARS-CoV-2 Entry by Using Human ACE2. Cell 2020, 181 (4), 894-904.e9.

23. Dassault Systèmes BIOVIA, Discovery Studio Modeling Environment, Release 2017, San Diego: Dassault Systèmes, 2016.

24. Morris, G. M.; Huey, R.; Lindstrom, W.; Sanner, M. F.; Belew, R. K.; Goodsell, D. S.; Olson, A. J., AutoDock4 and AutoDockTools4: Automated docking with selective receptor flexibility. $J$ Comput Chem 2009, 30 (16), 2785-2791.

25. Pettersen, E. F.; Goddard, T. D.; Huang, C. C.; Couch, G. S.; Greenblatt, D. M.; Meng, E. C.; Ferrin, T. E., UCSF Chimera--a visualization system for exploratory research and analysis. J Comput Chem 2004, 25 (13), 1605-12.

26. Trott, O.; Olson, A. J., AutoDock Vina: improving the speed and accuracy of docking with a new scoring function, efficient optimization, and multithreading. J Comput Chem 2010, 31 (2), 455461.

27. Humphrey, W.; Dalke, A.; Schulten, K., VMD: Visual molecular dynamics. Journal of Molecular Graphics 1996, 14 (1), 33-38. 
Tables:

Table 1. Binding affinities in $\mathrm{kcal} / \mathrm{mol}$ of five molecules on three different target proteins.

\begin{tabular}{|c|c|c|c|c|c|}
\hline $\begin{array}{l}\text { Binding Affinities } \\
\text { (kcal/mol) }\end{array}$ & Favipiravir & Hydroxychloroquine & Remdesivir & Lopinavir & Ritonavir \\
\hline SARS-CoV-2 ${ }^{\text {RBD }}$ & -5.1 & -4.2 & -4.9 & -6.9 & -6.4 \\
\hline SARS-CoV-2 Mpro-ac & -5.4 & -5.9 & -7.5 & -7.5 & -6.8 \\
\hline SARS-CoV-2 Mpro & -5.7 & -5.2 & -5.5 & -6.6 & -6.4 \\
\hline hFUR $^{\text {ac }}$ & -5.1 & -5.2 & -7.0 & -6.8 & -6.8 \\
\hline hFUR & -6.0 & -6.0 & -6.6 & -8.0 & -6.9 \\
\hline
\end{tabular}


Figures:

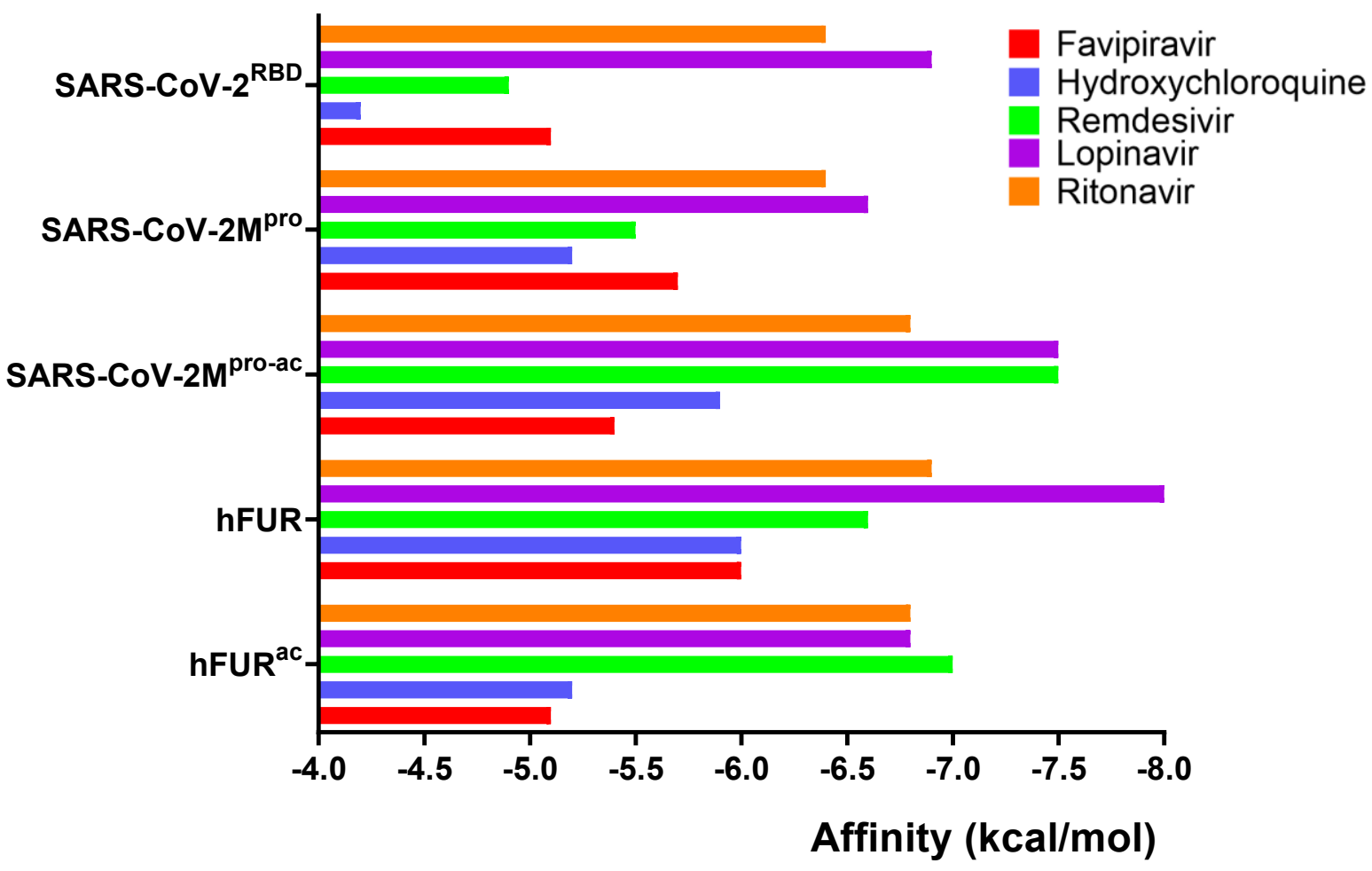

Figure 1. Graphical representation of binding affinities for five molecules on three proteins including the active site of proteases, SARS-CoV-2-Mro-ac and hFUR ${ }^{\text {ac. }}$. 


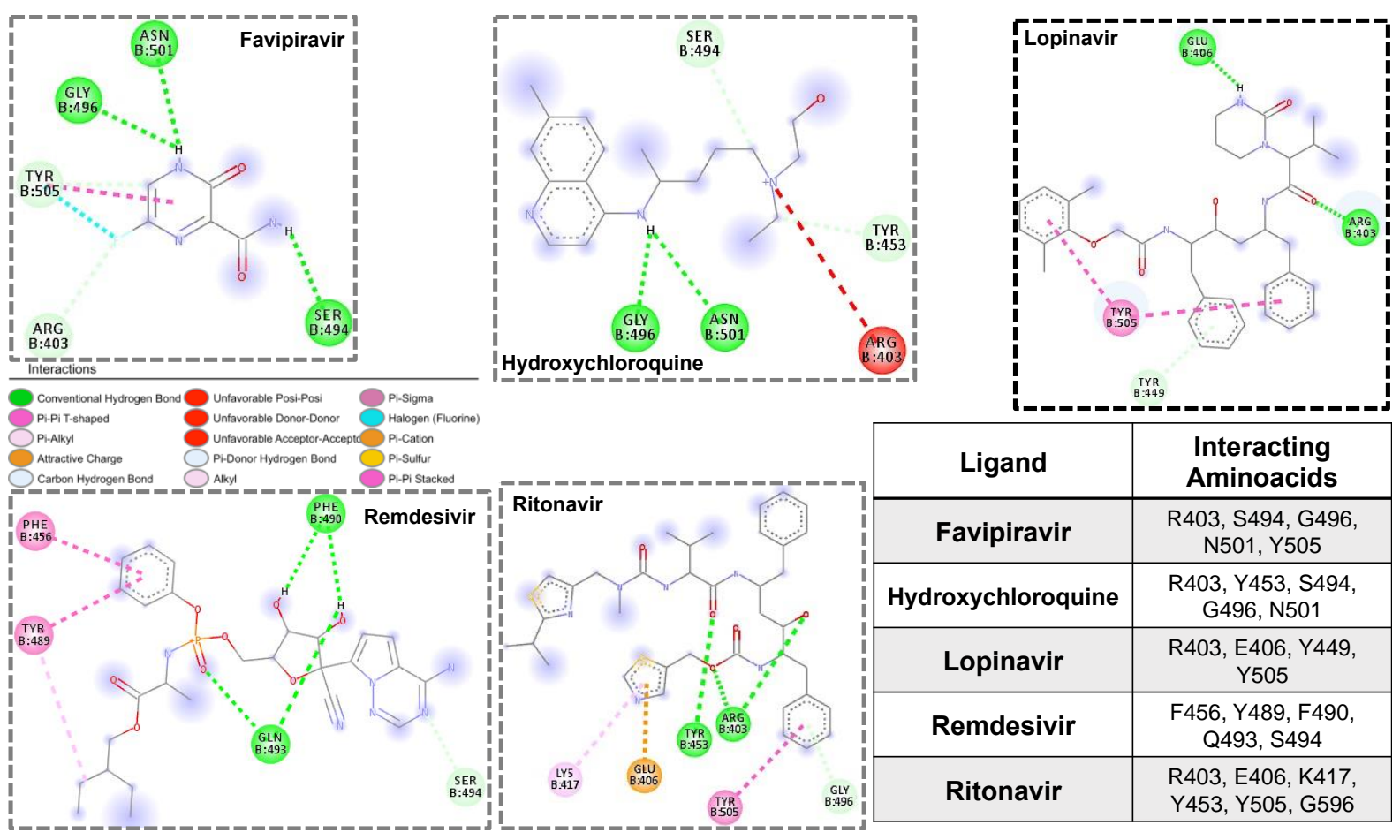

Figure 2. Molecular interactions for five molecules at the SARS-CoV-2RBD site. Drug molecule with the highest binding affinity was highlighted in bold frame. Legend for interactions was provided in the middle and interacting amino acid residues were provided in the table. 


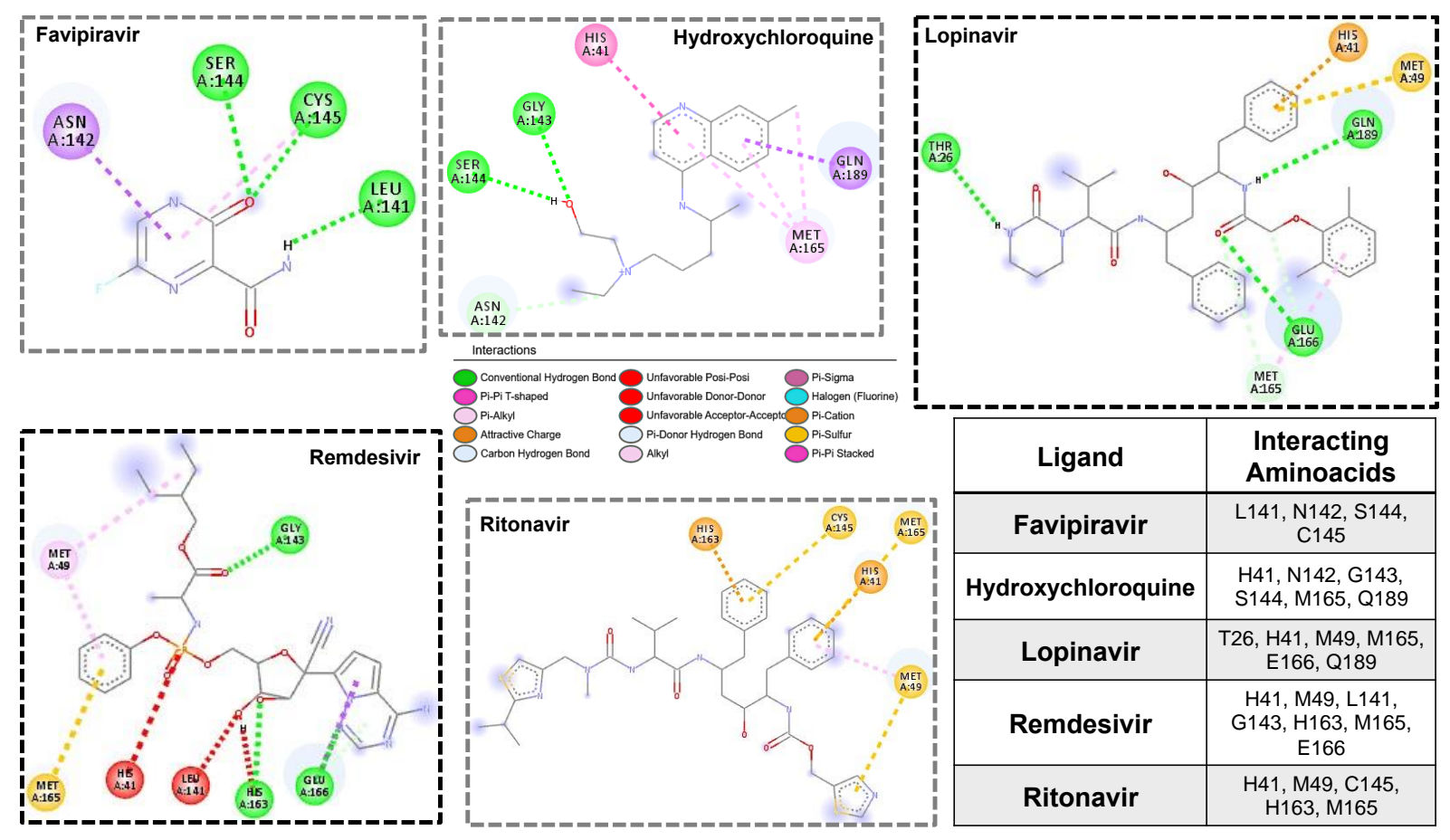

Figure 3. Molecular interactions for five molecules at the SARS-CoV-2 Mpro-ac $^{\text {pa }}$ site. Drug molecule with the highest binding affinity was highlighted in bold frame. Legend for interactions was provided in the middle and interacting amino acid residues were provided in the table. 


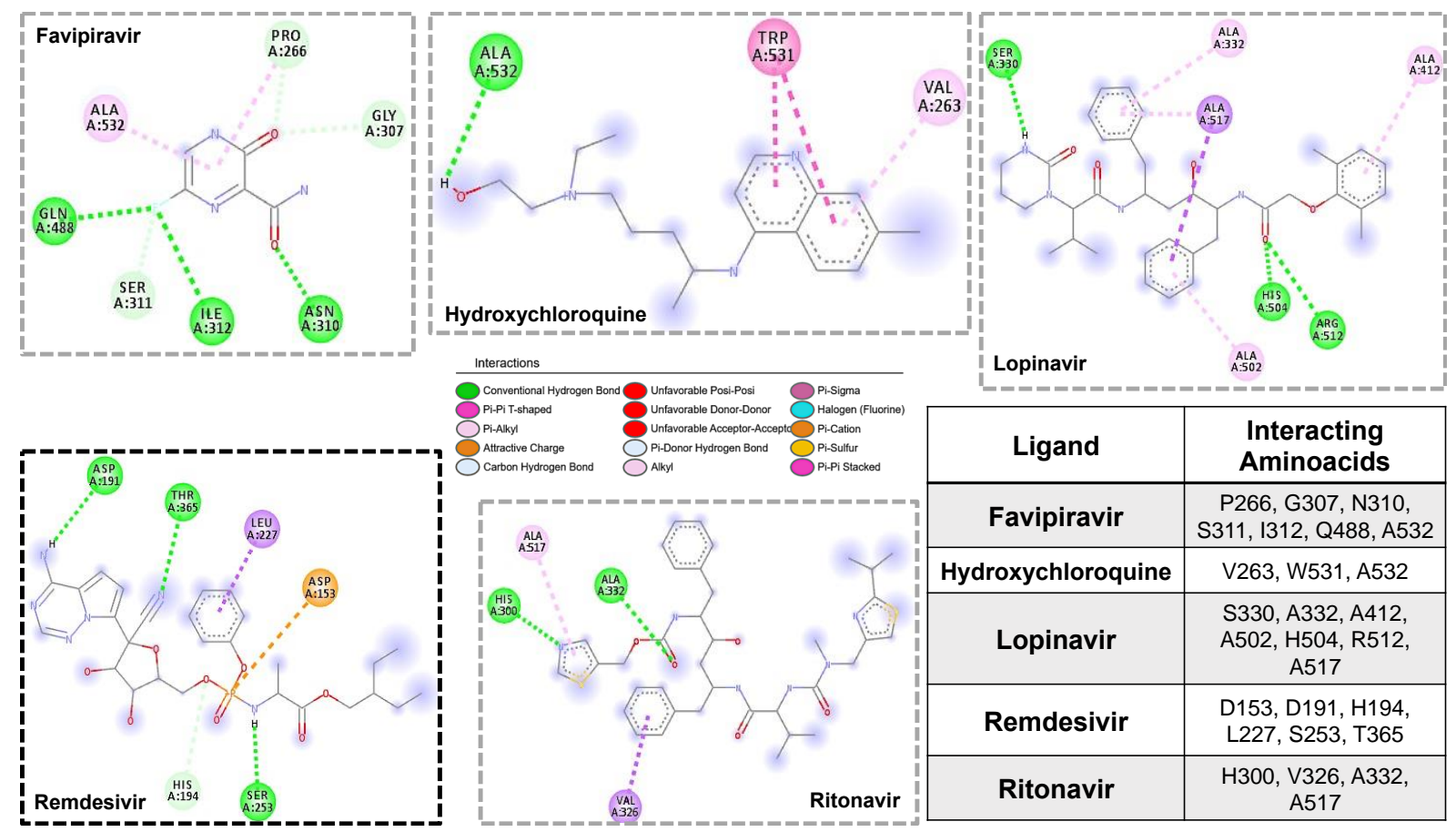

Figure 4. Molecular interactions for five molecules at the hFUR ${ }^{a c}$. Drug molecule with the highest binding affinity was highlighted in bold frame. Legend for interactions was provided in the middle and interacting amino acid residues were provided in the table. 
Supporting Information Figure:

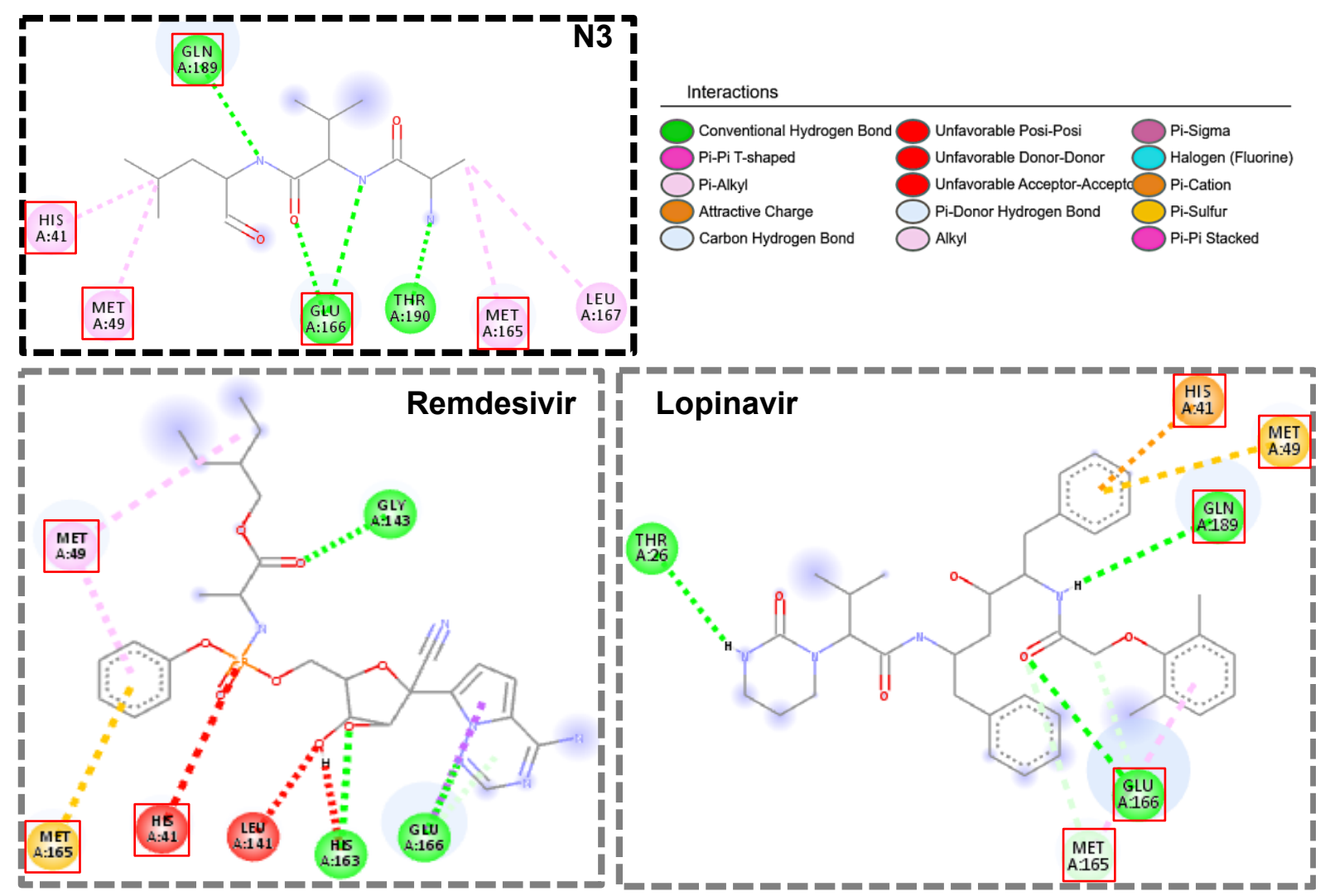

Figure SI-1. Comparison of N3 binding from crystal structure, PDB ID: 6LUV, and remdesivir and lopinavir binding from the current study at SARS-CoV-2

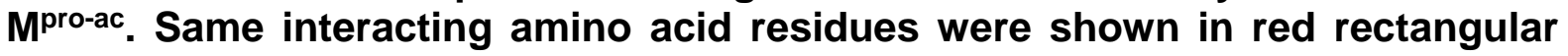
boxes. 


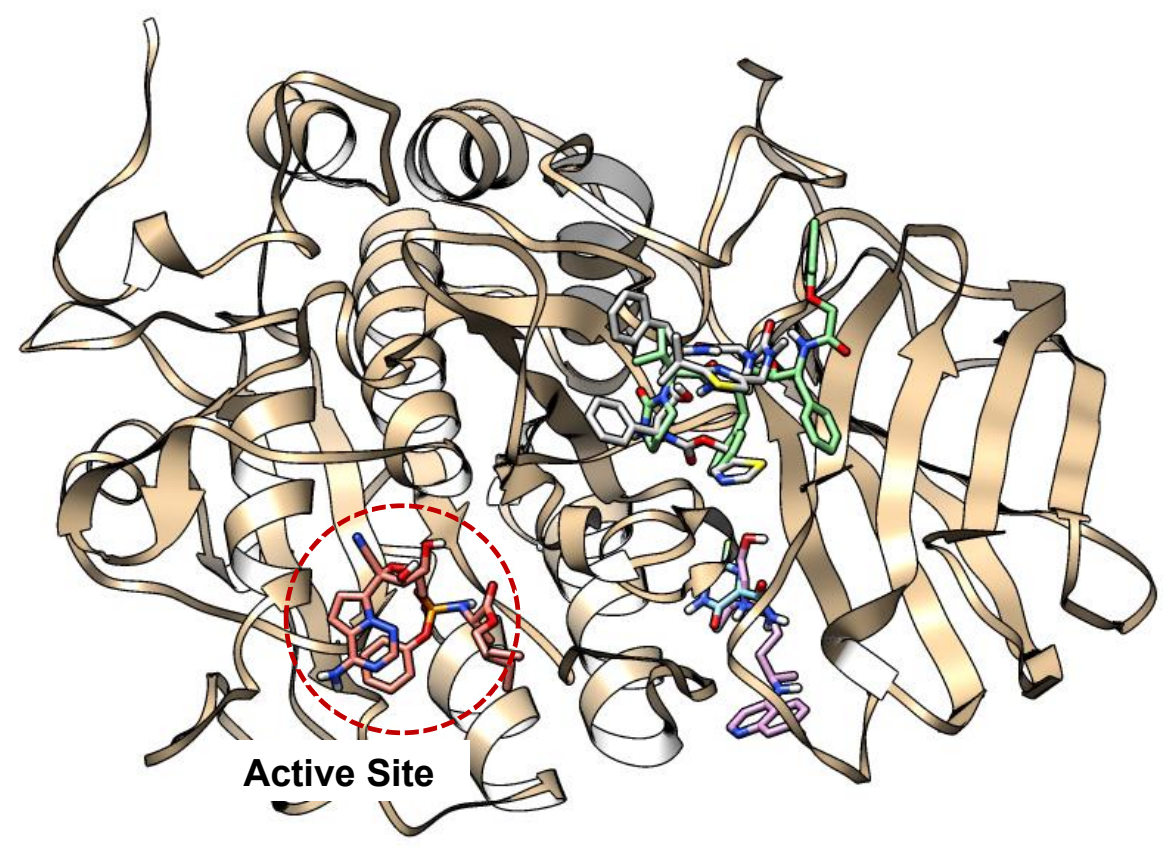

Figure SI-2. Molecular docking results for hFUR. Remdesivir was the only active molecule hitting the active site, although simulations grid box covered the entire protein. 

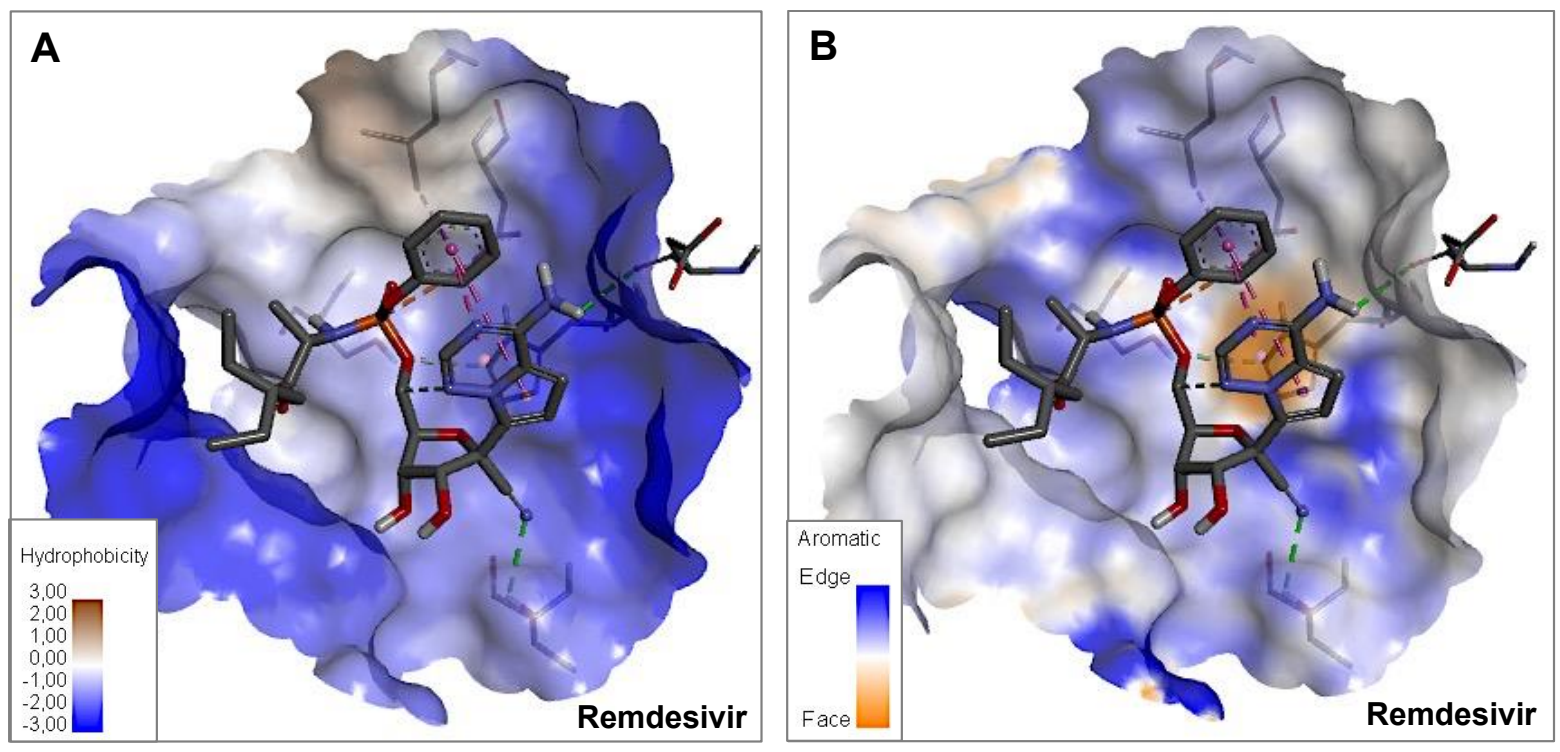

Figure SI-3. A) Hydrophobicity and B) aromaticity of hFUR ${ }^{\text {ac }}$. The active site is highly hydrophilic and aromatic, creating many face-to-face aromatic interactions with the aromatic rings of remdesivir 